\title{
Electronic health record phenotype in subjects with genetic variants associated with arrhythmogenic right ventricular cardiomyopathy: a study of 30,716 subjects with exome sequencing
}

\author{
Christopher M. Haggerty, $\mathrm{PhD}^{1}$, Cynthia A. James, ScM, PhD ${ }^{2}$, Hugh Calkins, MD², \\ Crystal Tichnell, MGC ${ }^{2}$, Joseph B. Leader, BA ${ }^{3}$, Dustin N. Hartzel, BS ${ }^{3}$, Christopher D. Nevius, BT $^{1}$, \\ Sarah A. Pendergrass, PhD ${ }^{3}$, Thomas N. Person, $\mathrm{MS}^{3}$, Marci Schwartz, ScM ${ }^{4}$, Marylyn D. Ritchie, $\mathrm{PhD}^{3}$, \\ David J. Carey, $\mathrm{PhD}^{5}$, David H. Ledbetter, $\mathrm{PhD}^{4}$, Marc S. Williams, MD ${ }^{4}$, Frederick E. Dewey, MD ${ }^{6}$, \\ Alexander Lopez, $\mathrm{MS}^{6}$, John Penn, $\mathrm{PSM}^{6}$, John D. Overton, $\mathrm{PhD}^{6}$, Jeffrey G. Reid, $\mathrm{PhD}^{6}$, \\ Matthew Lebo, $\mathrm{PhD}^{7,8}$, Heather Mason-Suares, $\mathrm{PhD}^{7,8}$, Christina Austin-Tse, $\mathrm{PhD}^{7}$, \\ Heidi L. Rehm, PhD ${ }^{7,8}$, Brian P. Delisle, PhD ${ }^{9}$, Daniel J. Makowski, DO ${ }^{10}$, Vishal C. Mehra, MD, PhD ${ }^{10}$, \\ Michael F. Murray, $\mathrm{MD}^{4}$ and Brandon K. Fornwalt, MD, PhD ${ }^{1}$
}

Purpose: Arrhythmogenic right ventricular cardiomyopathy (ARVC) is an inherited heart disease. Clinical follow-up of incidental findings in ARVC-associated genes is recommended. We aimed to determine the prevalence of disease thus ascertained.

Methods: Individuals ( $n=30,716)$ underwent exome sequencing. Variants in PKP2, DSG2, DSC2, DSP, JUP, TMEM43, or TGF 3 that were database-listed as pathogenic or likely pathogenic were identified and evidence-reviewed. For subjects with putative loss-offunction (pLOF) variants or variants of uncertain significance (VUS), electronic health records (EHR) were reviewed for ARVC diagnosis, diagnostic criteria, and International Classification of Diseases (ICD-9) codes.

Results: Eighteen subjects had pLOF variants; none of these had an EHR diagnosis of ARVC. Of 14 patients with an electrocardiogram, one had a minor diagnostic criterion; the rest were normal. A total of 184 subjects had VUS, none of whom had an ARVC diagnosis. The proportion of subjects with VUS with major (4\%) or minor (13\%) electrocardiogram diagnostic criteria did not differ from that of variant-negative controls. ICD-9 codes showed no difference in defibrillator use, electrophysiologic abnormalities or nonischemic cardiomyopathies in patients with pLOF or VUSs compared with controls.

Conclusion: pLOF variants in an unselected cohort were not associated with ARVC phenotypes based on EHR review. The negative predictive value of EHR review remains uncertain.

Genet Med advance online publication 4 May 2017

Key Words: arrhythmogenic right ventricular cardiomyopathy; electronic health record; exome sequencing; genotype-phenotype association; incidental findings

\section{INTRODUCTION}

Arrhythmogenic right ventricular cardiomyopathy (ARVC) is an inherited heart disease characterized by ventricular dysfunction and arrhythmias. ${ }^{1}$ ARVC has an estimated phenotypic prevalence of $1: 1000$ to $1: 5000,{ }^{2}$ and is among the leading causes of sudden cardiac death in people under 35 years of age-especially young athletes. ${ }^{3,4}$ Clinical symptoms are frequently absent before a sudden death event, ${ }^{5}$ so improved methods of screening and early diagnosis are needed to provide an opportunity for life-saving prophylaxis. ARVC is primarily attributed to genetic variants in cardiac desmosome genes, ${ }^{6,7}$ so a "genome-first" approach to patient identification is a promising option for earlier diagnosis. This paradigm is a natural extension of recommendations from the American College of Medical Genetics and Genomics that specific incidental findings from clinical genetic sequencing should be reported to patients because of the potential for medical benefit. ${ }^{8}$ Indeed, ARVC was one of the few selected conditions included with these guidelines, representing five of the 56 genes recommended for screening.

Recent studies have called into question the efficacy of incidental genetic findings for ARVC and other inherited

\footnotetext{
${ }^{1}$ Department of Imaging Science and Innovation, Geisinger Health System, Danville, Pennsylvania, USA; ${ }^{2}$ Division of Cardiology, Department of Medicine, Johns Hopkins University, Baltimore, Maryland, USA; ${ }^{3}$ Biomedical and Translational Informatics Institute, Geisinger Health System, Danville, Pennsylvania, USA; ${ }^{4}$ Genomic Medicine Institute, Geisinger Health System, Danville, Pennsylvania, USA; ${ }^{5}$ Weis Center for Health Research, Geisinger Health System, Danville, Pennsylvania, USA; ${ }^{6}$ Regeneron Genetics Center, Regeneron Pharmaceuticals, Tarrytown, New York, USA; ${ }^{7}$ Laboratory for Molecular Medicine, Partners HealthCare Personalized Medicine, Cambridge, Massachusetts, USA; ${ }^{8}$ Department of Pathology, Brigham and Women's Hospital, Harvard Medical School, Boston, Massachusetts, USA; ${ }^{9}$ Department of Physiology, University of Kentucky, Lexington, Kentucky, USA; ${ }^{10}$ Division of Cardiology, Geisinger Health System, Danville, Pennsylvania, USA. Correspondence: Christopher M. Haggerty (chrismhaggerty@gmail.com) 
cardiac diseases. ${ }^{9-13}$ Most notably, a study by Van Driest et al. ${ }^{11}$ concluded that there was no abnormal phenotype in individuals carrying potentially pathogenic variants for select genes associated with either long QT syndrome or Brugada syndrome, suggesting that notifying patients of incidental findings was unwarranted. While striking, this study was limited by the facts that only 2,022 individuals were studied, and only two genes-accounting for approximately $38 \%$ of long QT syndrome cases ${ }^{14}$ and $16 \%$ of Brugada syndrome cases $^{15}$-were screened. Moreover, the observed variants were almost exclusively missense or variants of uncertain significance (VUS). Missense variants have previously been shown to be both common ${ }^{10,16}$ and difficult to evaluate for pathogenicity, ${ }^{17}$ as is further evidenced by the lack of consensus among clinical laboratory reviews in the aforementioned study by Van Driest et al. ${ }^{11}$ Based on disease prevalence, ${ }^{11}$ the likelihood of identifying even one patient with a causal mutation linked to either syndrome in that cohort was only $34 \%$. Larger numbers are clearly needed to sufficiently power such analyses for rare diseases.

In this study, we used a 15 -fold larger cohort $(30,716$ subjects with exome sequencing), reviewed more genes (seven), and focused on "radical" putative loss-of-function (pLOF) variants, which have a higher probability of disease association, ${ }^{9}$ to provide a more comprehensive analysis of the genotypic prevalence of ARVC. Furthermore, since no other study has evaluated the phenotypes of individuals with pLOF ARVC variants ascertained through population sequencing, we reviewed electronic health records (EHR) for identified subjects to establish a genotype-phenotype association. While full disease penetrance was not expected, we hypothesized that a discernible EHR phenotype (such as the presence of ARVC diagnostic criteria, arrhythmias, or other primary cardiomyopathies) would be present in individuals with pLOF variants.

\section{Study information}

\section{MATERIALS AND METHODS}

The MyCode Community Health Initiative of Geisinger Health System (GHS) is an institutional review boardapproved research biorepository and precision medicine project. MyCode participants provide samples for research, including permission to link samples and associated data with information in their EHR. ${ }^{18}$ Through the "DiscovEHR" collaboration between GHS and the Regeneron Genetics Center, DNA samples from MyCode participants are used to generate exome sequence data to populate this database. ${ }^{18,19}$ Details of the exome sequencing and postprocessing have been described elsewhere ${ }^{19}$ (see the Supplementary Information online for additional detail). Briefly, sequencing was performed on an Illumina v4 HiSeq 2500 to a coverage depth such that over $95 \%$ of samples had greater than $85 \%$ of the target bases covered with a read depth greater than 20X. At the time of this study, the MyCode repository comprised 30,716 subjects of predominantly European ancestry. No exclusions were made for relatedness or ancestry.

\section{Variant evaluation}

We reviewed the ClinVar (http://ncbi.nlm.nih.gov/clinvar) and ARVC (http://arvcdatabase.info ${ }^{20}$ ) databases for all ARVC-associated variants in PKP2, DSP, DSC2, DSG2, JUP, TMEM43, and TGF 33 that were classified as pathogenic (P) or likely pathogenic (LP) as of 2 June 2015. The ClinVar search criteria included both the gene name and the term "arrhythmogenic right ventricular cardiomyopathy." For conflicting classifications, ClinVar superseded the ARVC database, and conflicting interpretations in ClinVar were resolved based on the most recent submission. The variants observed in the MyCode cohort were then further classified by expert review according to published methods ${ }^{21}$ consistent with the 2015 American College of Medical Genetics and Genomics-Association for Molecular Pathology guideline for sequence variant interpretation. ${ }^{22}$ Of note, loss-of-function is the predominant mechanism of pathogenicity for the desmosome genes in ARVC, ${ }^{9}$ so the evidence assertion for radical pLOF variants was generally very strong. This expert review was conducted by staff at the Laboratory for Molecular Medicine at Partners Personalized Medicine (C.A-T., H.M.-S., M.L.). To account for continuous, ongoing improvements in the bioinformatics pipeline with time, variant calls were rechecked against the most recent quality control-filtered data at the time of manuscript preparation. This pipeline included GATK best practices for variant calling, ${ }^{23}$ and filtering with GATK for genotype quality with a threshold of 20 .

\section{Subject identification and phenotype analysis}

All subjects carrying at least one potential ARVC variant (database reported "P/LP") were identified. Age ( \pm 5 years) and sex-matched subjects (5:1 match) were randomly ascertained from MyCode subjects lacking ARVC-associated variants.

For all subjects with potential variants and a random subset $(10 \%)$ of controls, we completed a blinded expert review of the primary data, including:

1. Most recent nonpaced electrocardiogram (ECG) (initial review by C.A.J. and C.T.; any abnormal finding read by H.C. for classification).

2. Most recent echocardiogram for which the right ventricular function and size were not explicitly reported as normal (V.C.M. and D.J.M.). A random $20 \%$ of the "normal" cases were also reviewed to ensure accuracy.

Data from the most recent Holter monitoring were also reviewed. Findings were compared against the diagnostic Task Force criteria for ARVC, which include right ventricular dysfunction and depolarization/repolarization abnormalities on ECG. ${ }^{24}$ International Classification of Diseases, Ninth Revision (ICD-9) codes in patient records as of 21 July 2015 were reviewed for specific (see Supplementary Table S1) and nonspecific (Supplementary Table S2) codes. As appropriate, the cause of death (i.e., cardiac, noncardiac, or unknown) was 
assessed from a blinded physician chart review (B.K.F.) of death certificates or contemporaneous physician notes.

Separately, the GHS EHR was reviewed for individuals who had experienced any medical encounter with an associated diagnosis of arrhythmogenic right ventricular cardiomyopathy or dysplasia (Supplementary Table S1). A physician chart review (B.K.F.) of the two most recent cardiology, internal/family medicine, and discharge notes (or any two subspecialty notes when other notes were not available) was performed for affirmative documentation of disease. This blinded procedure was also used to confirm the diagnostic status for a random subset (33\%) of subjects with potential variants, plus any subject with an external cardiology referral.

\section{Statistical analysis}

Statistical testing was performed using $\mathrm{R}$ (version 3.3.2). Descriptive statistics are reported as mean \pm standard deviation. Group differences in phenotypes were compared using Fisher's exact tests; the log-rank test was used to compare survival, using the OIsurv package in $\mathrm{R}^{25}$ Reported $P$-values were not adjusted for multiple comparisons.

\section{RESULTS}

\section{Missense variants are common; previously reported pLOF} variants are rare

A total of 323 rare variants were database-listed as "P/LP" in ClinVar and/or the ARVC database (see Supplementary Table S3). Of these, 45 potentially pathogenic variants were identified in 301 MyCode participants. One variant (TMEM43 c. $705+7 \mathrm{G}>$ A) was observed in 76 subjects and immediately reclassified as likely benign due to the high allele frequency $(>0.075 \%) .{ }^{21}$ Furthermore, the variant calls for seven subjects did not pass genotype quality filtering, and were excluded. The remaining 215 patients $(0.7 \%$ of the population) comprised the potentially pathogenic variant group (Figure 1; Table 1). The mean age was $62 \pm 17$ years. The distribution with respect to affected gene is shown in Supplementary Table S4. Missense variants were most prevalent with respect to the observed variants (30 of 44 (excluding TMEM43 c.705+7G > A); 68\%) and the number of subjects (191 of $215 ; 89 \%$ ).

After evidence review, nine radical pLOF variants (nonsense and splice site) met the criteria to be classified as P/LP. Supporting evidence for other variants, including all missense variants, was insufficient and they were scored as VUS or likely benign (Table 1). Of note, eight of the nine pLOF variants were documented in the ClinVar database; only one of the 32 observed variants unique to the ARVC database was confirmed.

The nine pLOF variants were identified in 18 subjects (1:1706). The mean age of this group was $59 \pm 18$ years (Table 2), and the mean body mass index (BMI) was $32 \pm 8$.

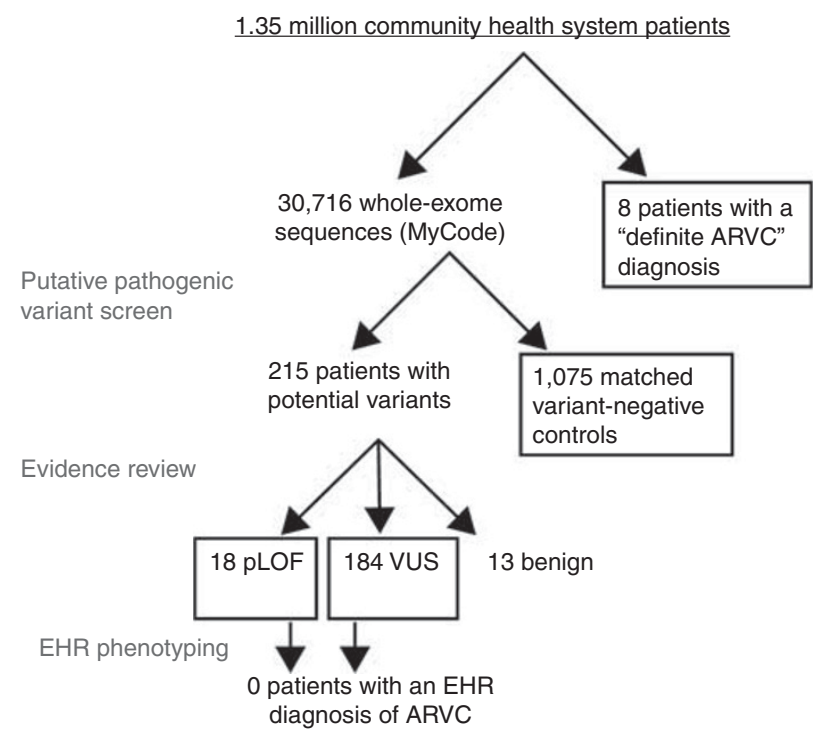

Figure 1 Overview of the study design and patient group identities. ARVC, arrhythmogenic right ventricular cardiomyopathy; EHR, electronic health records; pLOF, putative loss of function; VUS, variant of uncertain significance.

No increase in the prevalence of ARVC diagnostic criteria in patients with pLOF variants

The 18 subjects with pLOF variants had a median of 9.5 years of EHR data (range: 0-16 years). Based on ICD-9 coding and a randomized chart review, none had a documented diagnosis of ARVC. Fourteen subjects (78\%) had a previous ECG. These were manually reviewed for diagnostic depolarization/repolarization criteria (Table 3 ). One of the 14 subjects satisfied a minor criterion (inverted $\mathrm{T}$-waves in $\mathrm{V}_{5}$ and $\mathrm{V}_{6}$ ), which qualifies for a "borderline" diagnosis with the addition of the LP variant. Separately, echocardiograms were reviewed for the eight subjects (44\%) with previous studies, and right ventricular function was reported as "normal" for all. Four of the 18 subjects with pLOF variants (22\%) had neither an ECG nor echocardiogram-including three individuals younger than 37 years of age.

Given the uncertain importance of a rare VUS in ARVCassociated genes, primary EHR data for the 184 individuals with such variants were also reviewed (Table 2). The average BMI for these subjects was $31 \pm 7$. Based on ICD-9 coding and a randomized chart review, none of these individuals had a documented diagnosis of ARVC. ECGs were available for 160 individuals (87\%); 83\% satisfied no diagnostic criteria, 4\% satisfied a major criterion, and $13 \%$ satisfied a minor criterion (Table 3). These frequencies were comparable to those of the variant-negative control group, from which $5 \%$ and $17 \%$ were found to have major and minor ECG criteria, respectively. Hence, the findings in the VUS group were statistically similar to the variant-negative control group $(P=0.54$ and $P=0.35$, respectively). The same trends were observed with respect to echocardiographic and Holter monitoring criteria (Table 3). 
Table 1 Details of variants database listed as "P/LP" and identified in 30,716 patients

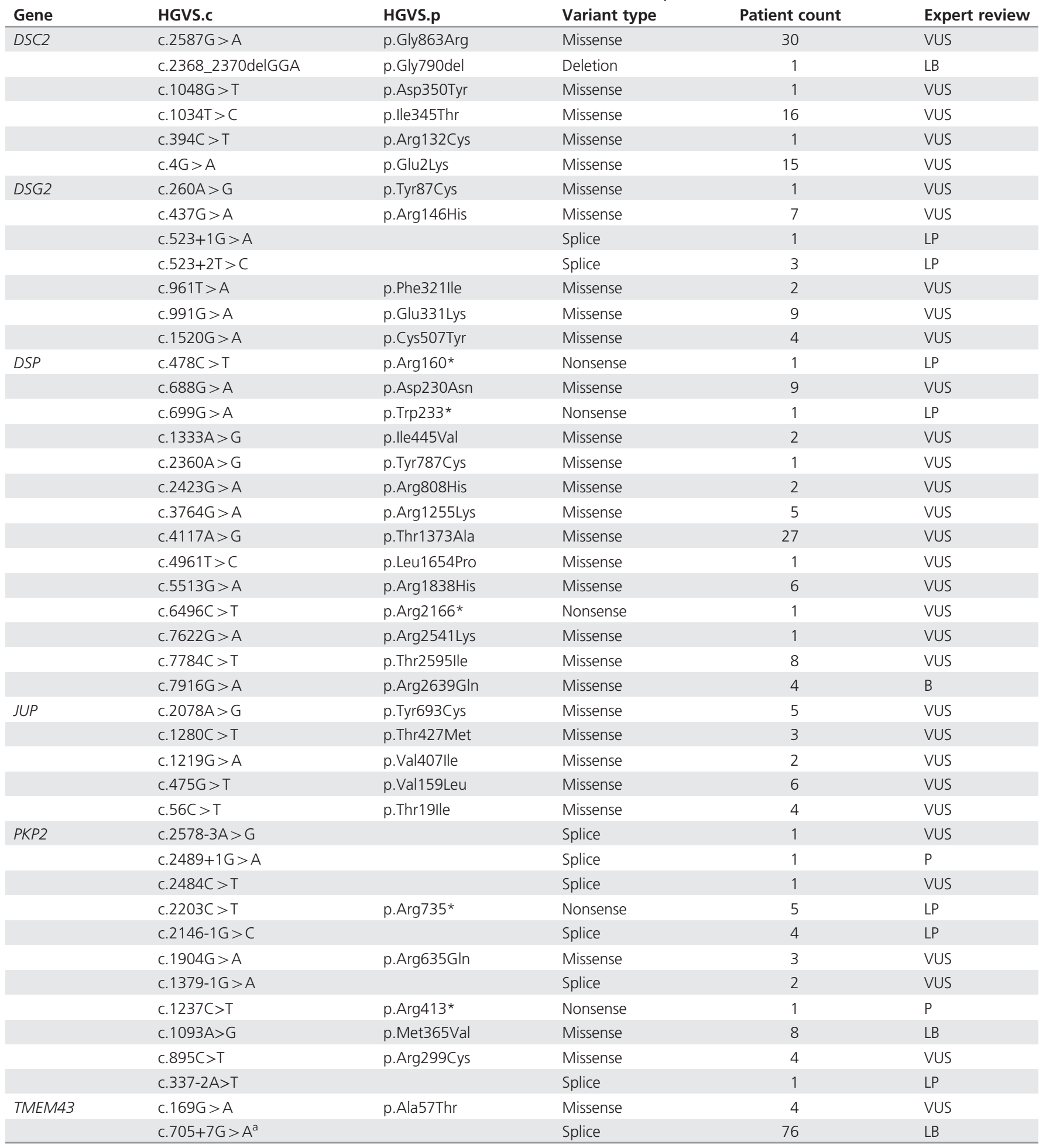

B, benign; HGVS.C, Human Genome Variation Society CDNA change; HGVS.p, Human Genome Variation Society amino acid change; LB, likely benign; LP, likely pathogenic; P, pathogenic; VUS, variant of uncertain significance.

a Reclassified as likely benign based on observed allele frequency

Nonspecific phenotypes distinguish known ARVC cases but not potential variant groups

Thirty patients with at least one instance of an ARVC diagnostic code in their EHR were identified, out of 1.35 million subjects. A physician chart review confirmed an ARVC diagnosis in only 8 subjects (1:168,750; Table 2); diagnostic codes were determined to be inappropriately applied in the remaining 22 subjects. No exome sequence data were available for these individuals. 
Table 2 Patient demographic details by group

\begin{tabular}{lccc} 
& Number of patients & Age (mean \pm standard deviation; in years) & Sex (\% female) \\
\hline pLOF variant & 18 & $59 \pm 18$ & 56 \\
VUS & 184 & $62 \pm 17$ & 58 \\
Variant-negative control & 1,075 & $61 \pm 17$ & 58 \\
Definite ARVC & 8 & $41 \pm 16$ & 63 \\
\hline
\end{tabular}

ARVC, arrhythmogenic right ventricular cardiomyopathy; pLOF, putative loss of function; VUS, variant of uncertain significance.

Table 3 Summary findings of 2010 Task Force criteria by group

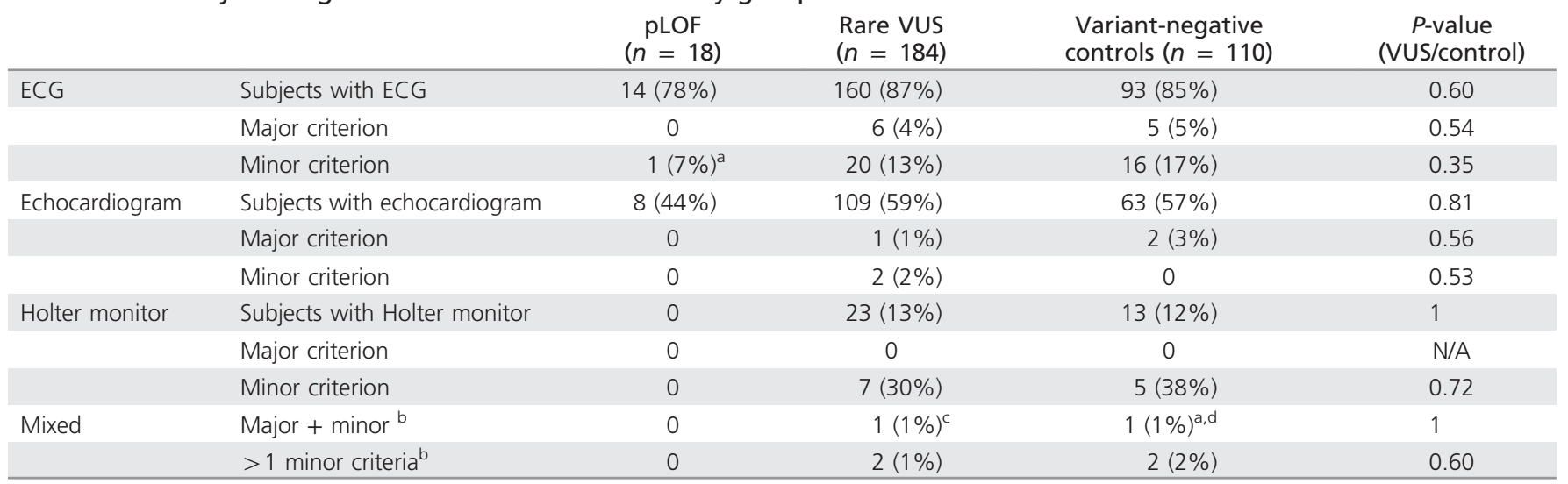

ECG, Electrocardiogram; pLOF, Putative loss of function; VUS, Variant of uncertain significance.

Data presented as number (percentage).

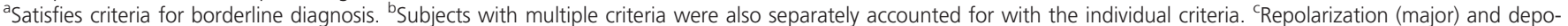

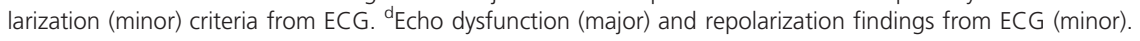

Nonspecific ICD-9 composite phenotypes-nonischemic cardiomyopathies, cardiac electrophysiologic abnormalities, ARVC "characteristic symptoms," and automatic implantable cardioverter defibrillator use-for the pLOF, VUS, variantnegative controls, and definite ARVC study groups are compared in Figure 2a. The prevalence of nonischemic cardiomyopathies, electrophysiologic abnormalities, and automatic implantable cardioverter defibrillator use was significantly higher in the definite ARVC group, whereas the patients with a pLOF variant or VUS showed prevalences similar to those in the negative control group.

\section{Increased all-cause mortality in the pLOF group}

We compared all-cause mortality (age at death) between groups (pLOF, VUS, and variant-negative controls). For the control and VUS groups, $89 \%$ and $92 \%$ of subjects were alive at the time of analysis, respectively $(P=0.19$; Figure $2 \mathbf{b})$. By comparison, only $72 \%$ of subjects (13 of 18 ) in the pLOF group were alive, representing significantly increased mortality compared with the VUS group $(P=0.003)$. However, from the chart review, the causes of death for the five pLOF subjects were non-cardiac related in four cases and unknown in one.

\section{DISCUSSION}

Our major findings appear to parallel recent studies in cardiac genetics with respect to the high prevalence of rare variants once thought to be disease-causing, but weak association with classic disease symptoms. ${ }^{10-13,16}$ However, the present work specific to ARVC represents a significant advance for several reasons. This study represents the largest analysis to date of next-generation sequencing data to evaluate the presence of putative genetic variants related to ARVC. The size and scope of this study (inclusive of all primary ARVC genes) provided a 98 to $99 \%$ likelihood of finding at least one subject with ARVC and a causal variant. This high probability contrasts with previous studies, such as the work by Van Driest et al., ${ }^{11}$ which have been drastically underpowered to study rare diseases and their associated radical genetic variants. ${ }^{11}$ Indeed, we observed previously reported pLOF variants in 18 (1:1706) individuals, which were further adjudicated as P/LP following expert evidence review. This study also represents the first population-based analysis of ARVC genetics with linked EHR data to evaluate genotype-phenotype associations. With these linked data, we reviewed diagnostic codes for all subjects, ECGs for $86 \%$ of the pLOF and rare VUS groups, and right ventricular function via echocardiogram in $58 \%$. Our findings suggest that, in unselected individuals with incidentally detected pLOF variants, genetic penetrance may be much lower than estimates (40-60\%) from familial studies ${ }^{5}$ suggest, as no individual had a documented diagnosis of disease, and only one subject satisfied any additional minor diagnostic criteria. Moreover, our findings demonstrate that individuals with a rare VUS also have an unremarkable 


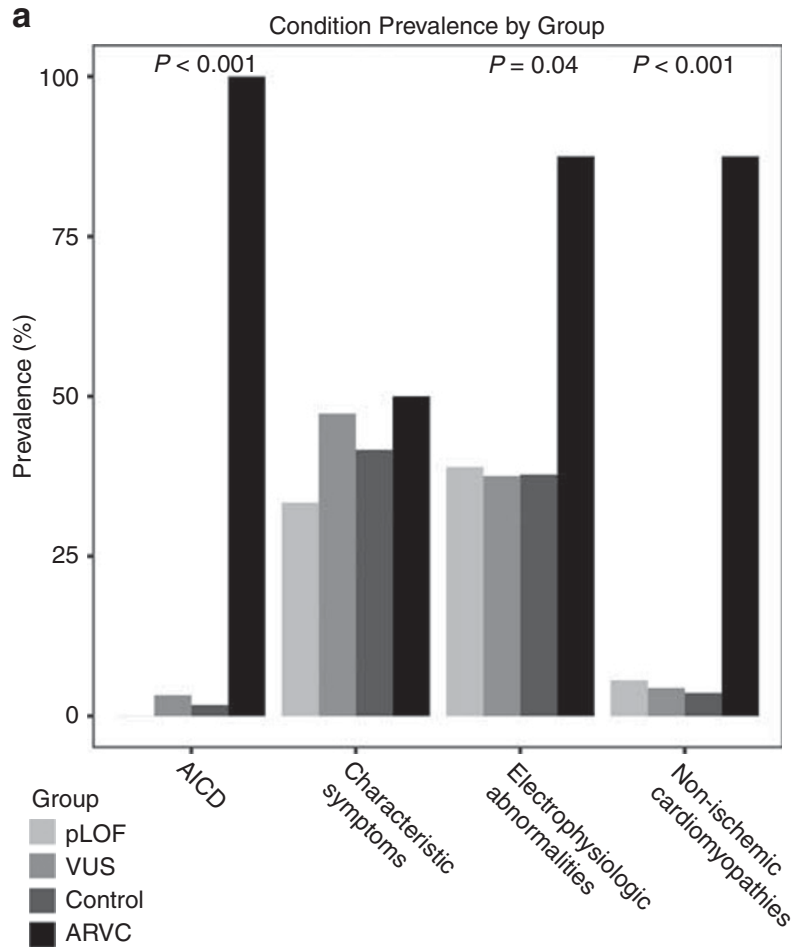

b

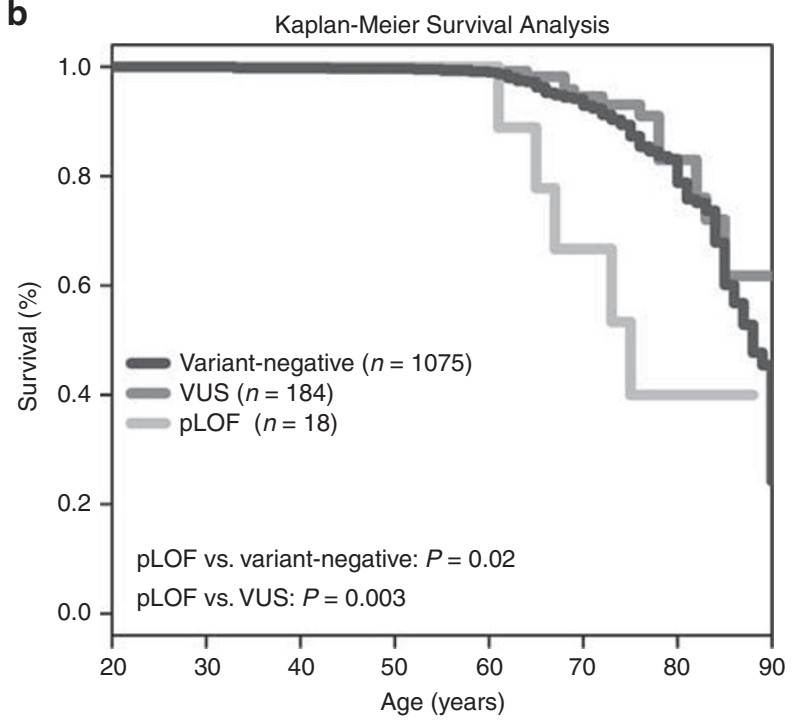

Figure 2 Condition prevalence and survival analysis. (a) Prevalence of the composite ICD-9 categories within each study group. P-values denote Fisher's exact test for a given category. (b) Kaplan-Meier survival estimates for each study group, showing that the pLOF group had a significantly reduced survival by log-rank test. AICD, automatic implantable cardioverter defibrillator; ARVC, arrhythmogenic right ventricular cardiomyopathy; pLOF, putative loss of function; VUS, variant of uncertain significance.

phenotype, with no existing diagnoses and diagnostic criteria frequencies in line with the observed false-positive rate in variant-negative controls. Several studies have documented the considerable background noise in our understanding of putative pathogenic ARVC variants, ${ }^{9,10}$ but none has previously demonstrated that this noise has no phenotypic consequence.

Vigorous exercise is the most significant known modulator of genetic penetrance for ARVC. ${ }^{26,27}$ In fact, current guidelines restrict athletic participation following even a possible diagnosis. ${ }^{28}$ Considering the obese average BMI of our cohort (greater than 32 in the pLOF group), it is likely that few subjects, if any, regularly participate in the level of vigorous physical activity typically associated with ARVC. Such a lifestyle likely confers a protective effect in the setting of genetic predisposition to ARVC and may have played a role in the low penetrance we observed in our study.

While our phenotype determination was not made from prospective patient evaluations, $86 \%$ of the individuals studied had a nonpaced ECG available for review. ECG abnormalities comprise two of the six elements of ARVC diagnosis, and the negative predictive value of a normal ECG is very high, as past studies have shown that over $80 \%$ of diagnosed ARVC patients have ECG abnormalities. ${ }^{29,30}$ Hence, while the available data are insufficient to definitively determine the status for the $13 \%$ of subjects with observed abnormalities or the $14 \%$ without ECGs, there is a high degree of certainty that the $73 \%$ of subjects whose ECG did not satisfy Task Force criteria do not have ARVC.

These results are not an inherent indictment of clinical follow-up for incidental genetic findings of ARVC, as there was at least one case of suggestive findings discovered through a genome-first ascertainment. The increased all-cause mortality in the small group of subjects with pLOF variants was also suggestive of a meaningful finding, although confirmation with a larger sample size is necessary. Instead, our findings strongly indicate that such efforts should be managed conservatively and with stringent evaluation of observed genetic variants based on current standards. Even for individuals identified with radical pLOF variants through genome-first ascertainment, low penetrance should be assumed because such screening is likely to maximize the probability of false-positive findings. Furthermore, this low penetrance may be exacerbated in the absence of environmental or lifestyle factors, such as a vigorously active lifestyle. Thus, the incidental identification of a pathogenic variant does not appear to warrant automatic acceptance as a major criterion for ARVC diagnosis, as it is for patients ascertained through clinical presentation or familial testing. ${ }^{24}$ Similar modification of family history criteria for arrhythmic risk stratification has recently been proposed. ${ }^{30}$ Ultimately, more research is needed to develop evidence-based guidelines for the clinical management of patients with incidentally identified pLOF variants in ARVC-associated genes.

\section{Other potential mechanisms to explain the results Genetic modulation of penetrance}

Additional unknown genetic factors may also explain the low penetrance we observed. For example, there has been some debate about the importance of compound or digenic heterozygosity to ARVC, ${ }^{9,31,32}$ which may predispose patients 
to a more severe phenotype. ${ }^{5,32}$ Furthermore, since only 50 to $70 \%$ of diagnosed cases have been attributed to a genetic cause, unrecognized genes may play critical roles. More extensive use of next-generation sequencing may help to address such questions. ${ }^{33}$ None of the subjects in our cohort had more than one known variant, although potential novel variants were not assessed.

\section{Phenotype misclassification}

ARVC is a relatively new and evolving clinical entity that may be underrecognized in clinical populations. The observed prevalence of diagnosed ARVC in our large community health system was $1: 168,750$, which is two orders of magnitude below reported prevalence and supports this assertion. Since our evaluation was based on EHR data, we cannot rule out clinical phenotype misclassification in the $27 \%$ of our cohort who did not have a documented normal ECG. Moreover, without a formalized set of evidence-based diagnostic criteria for the expanding phenotypic spectrum of ARVC (e.g., bi-ventricular and left-dominant forms), ${ }^{34}$ our ability to rule out these less common manifestations of the disease from this cohort is limited.

\section{Sampling bias}

Given the advanced mean age of the sequenced cohort, it is possible that sampling was biased for survivors. Since ARVC often presents with sudden death in early adulthood, truly affected individuals may have died at a younger age and are therefore not represented in the data. Furthermore, the presence of other age-related comorbidities, such as coronary artery disease, in this older cohort may also contribute to an underdiagnosis of ARVC. Future studies should seek to incorporate younger individuals into penetrance estimates.

\section{Limitations}

This study was retrospective and used data derived from our institutional EHR, which had a median of 12 years of longitudinal data per patient for the entire MyCode cohort. ${ }^{18}$ Prospective targeted phenotyping of pLOF subjects is forthcoming through our institution's GenomeFIRST return of results initiative, which will provide additional information on genotype-phenotype relationships and better clarify the predictive value of EHR phenotyping. ${ }^{35}$ However, the only other study using next-generation sequencing to study ARVC variants in 6,354 unselected individuals did not have linked EHR data. ${ }^{10}$ With a fivefold larger sequenced population and linkage to EHR, our study offers substantially more comprehensive results.

ARVC exhibits age-dependent penetrance, with symptoms and diagnostic criteria developing with time. ${ }^{30}$ Therefore, without completed lifelong follow-up, we cannot definitively say that none of the subjects in our cohort will develop new symptoms. However, previous studies have reported that the cumulative prevalence of ARVC is essentially flat after 60 years of age, ${ }^{36}$ suggesting that few of our subjects could be expected to develop new symptoms, given the mean age of the cohort.

We did not evaluate novel variants, instead focusing on previously observed variants. This was a similar approach to the recent work by Ghouse et al. ${ }^{12,13}$ The addition of novel pLOF variants will increase the likelihood of identifying affected individuals, but will also increase the genotype prevalence estimates. Future studies will include potentially novel variants.

\section{CONCLUSION}

In a large, unselected cohort with exome sequencing data linked to EHR, rare variants listed as P/LP in database resources are common (1:143 genotypic prevalence); however, only $20 \%$ of the observed variants ( 9 of 44 ) were pLOF. Of the 18 individuals (1:1706) identified with a pLOF variant, the majority (72\%) had a normal ECG and were likely unaffected, while only $6 \%$ (one subject) satisfied a minor ARVC diagnostic criterion. Because of the importance of environmental factors on ARVC pathogenesis, identification of radical variants alone does not provide a genome-first solution to the identification of ARVC. A conservative approach to clinical return of incidental genetic findings associated with ARVC with further in-person condition-specific phenotyping is warranted.

\section{SUPPLEMENTARY MATERIAL}

Supplementary material is linked to the online version of the paper at http://www.nature.com/gim

\section{ACKNOWLEDGMENTS}

This work was supported in part by the Regeneron Genetics Center, Geisinger Health System, and the National Institutes of Health (grant DP5 OD012132). We acknowledge all participants in the GHS MyCode Community Health Initiative.

\section{DISCLOSURE}

Lebo, Mason-Suares, Austin-Tse, and Rehm work for a fee-forservice laboratory to perform clinical genetic testing. Murray has consulted for Invitae and Merck. Calkins has received research support from Medtronic and St. Jude Medical. The other authors declare no conflict of interest.

\section{REFERENCES}

1. Marcus Fl, Fontaine GH, Guiraudon G, et al. Right ventricular dysplasia: a report of 24 adult cases. Circulation 1982;65:384-398.

2. Basso C, Corrado D, Marcus Fl, Nava A, Thiene G. Arrhythmogenic right ventricular cardiomyopathy. Lancet 2009;373:1289-1300.

3. Corrado D, Basso C, Schiavon M, Thiene G. Screening for hypertrophic cardiomyopathy in young athletes. N Engl J Med 1998;339:364-369.

4. Thiene G, Nava A, Corrado D, Rossi L, Pennelli N. Right ventricular cardiomyopathy and sudden death in young people. N Engl J Med 1988;318:129-133.

5. Groeneweg JA, Bhonsale A, James CA, et al. Clinical presentation, longterm follow-up, and outcomes of 1001 arrhythmogenic right ventricular dysplasia/cardiomyopathy patients and family members. Circ Cardiovasc Genet 2015;8:437-446.

6. McKoy G, Protonotarios N, Crosby A, et al. Identification of a deletion in plakoglobin in arrhythmogenic right ventricular cardiomyopathy with palmoplantar keratoderma and woolly hair (Naxos disease). Lancet 2000;355:2119-2124. 


\section{ORIGINAL RESEARCH ARTICLE}

7. Campuzano O, Alcalde M, Allegue C, et al. Genetics of arrhythmogenic right ventricular cardiomyopathy. J Med Genet 2013;50:280-289.

8. Green RC, Berg JS, Grody WW, et al. ACMG recommendations for reporting of incidental findings in clinical exome and genome sequencing. Genet Med 2013;15:565-574

9. Kapplinger JD, Landstrom AP, Salisbury BA, et al. Distinguishing arrhythmogenic right ventricular cardiomyopathy/dysplasia- associated mutations from background genetic noise. J Am Coll Cardiol 2011;57: 2317-2327.

10. Andreasen C, Nielsen JB, Refsgaard L, et al. New population-based exome data are questioning the pathogenicity of previously cardiomyopathy-associated genetic variants. Eur J Hum Genet 2013;21: 918-928.

11. Van Driest SL, Wells QS, Stallings S, et al. Association of arrhythmiarelated genetic variants with phenotypes documented in electronic medical records. JAMA 2016;315:47-57.

12. Ghouse J, Have CT, Skov MW, et al. Numerous Brugada syndromeassociated genetic variants have no effect on J-point elevation, syncope susceptibility, malignant cardiac arrhythmia, and all-cause mortality. Genet Med, e-pub ahead of print 6 October 2016.

13. Ghouse J, Have CT, Weeke $P$, et al. Rare genetic variants previously associated with congenital forms of long QT syndrome have little or no effect on the QT interval. Eur Heart J 2015;36:2523-2529.

14. Goldenberg I, Zareba W, Moss AJ. Long QT syndrome. Curr Prob/ Cardiol 2008;33:629-694.

15. Crotti L, Marcou CA, Tester DJ, et al. Spectrum and prevalence of mutations involving BrS1- through BrS12-susceptibility genes in a cohort of unrelated patients referred for Brugada syndrome genetic testing: implications for genetic testing. J Am Coll Cardiol 2012;60:1410-1418.

16. Bick AG, Flannick J, Ito $K$, et al. Burden of rare sarcomere gene variants in the Framingham and Jackson Heart Study cohorts. Am J Hum Genet 2012;91:513-519.

17. Kapa S, Tester DJ, Salisbury BA, et al. Genetic testing for long-QT syndrome: distinguishing pathogenic mutations from benign variants. Circulation 2009;120:1752-1760

18. Carey DJ, Fetterolf SN, Davis FD, et al. The Geisinger MyCode community health initiative: an electronic health record-linked biobank for precision medicine research. Genet Med 2016;18:906-913.

19. Dewey FE, Gusarova V, O'Dushlaine C, et al. Inactivating variants in ANGPTL4 and risk of coronary artery disease. N Engl J Med 2016;374: 1123-1133.

20. Lazzarini $E$, Jongbloed JDH, Pilichou $K$, et al. The ARVD/C genetic variants database: 2014 update. Hum Mutat 2015;36:403-410.

21. Duzkale $H$, Shen J, Mclaughlin $H$, et al. A systematic approach to assessing the clinical significance of genetic variants. Clin Genet 2013;84: 453-463.

22. Richards S, Aziz N, Bale S, et al. Standards and guidelines for the interpretation of sequence variants: a joint consensus recommendation of the American College of Medical Genetics and Genomics and the Association for Molecular Pathology. Genet Med 2015;17:405-424.

23. GATKIBest Practices. https://software.broadinstitute.org/gatk/best-practices/. Accessed 30 January 2017

24. Marcus Fl, McKenna WJ, Sherrill D, et al. Diagnosis of arrhythmogenic right ventricular cardiomyopathy/dysplasia. Eur Heart J 2010;31:806-814.

25. Diez DM. Olsurv: Survival analysis supplement to OpenIntro guide. $R$ package version 0.2 https://cran.r-project.org/package = Olsurv. Accessed 1 February, 2017.

26. Ruwald AC, Marcus F, Estes NAM, et al. Association of competitive and recreational sport participation with cardiac events in patients with arrhythmogenic right ventricular cardiomyopathy: results from the North American multidisciplinary study of arrhythmogenic right ventricular cardiomyopath. Eur Heart J 2015;36:1735-1743.

27. James CA, Bhonsale A, Tichnell $C$, et al. Exercise increases age-related penetrance and arrhythmic risk in arrhythmogenic right ventricular dysplasia/cardiomyopathy-associated desmosomal mutation carriers. J Am Coll Cardiol 2013;62:1290-1297.

28. Maron BJ, Udelson JE, Bonow RO, et al. Eligibility and disqualification recommendations for competitive athletes with cardiovascular abnormalities: Task Force 3: hypertrophic cardiomyopathy, arrhythmogenic right ventricular cardiomyopathy and other cardiomyopathies, and myocarditis. J Am Coll Cardiol 2015:66:2362-2371.

29. te Riele AS, James CA, Bhonsale $A$, et al. Malignant arrhythmogenic right ventricular dysplasia/cardiomyopathy with a normal 12-lead electrocardiogram: a rare but underrecognized clinical entity. Heart Rhythm 2013;10:1484-1491.

30. te Riele AS, James CA, Groeneweg JA, et al. Approach to family screening in arrhythmogenic right ventricular dysplasia/cardiomyopathy. Eur Heart J 2016:37:755-763.

31. Xu T, Yang Z, Vatta M, et al. Compound and digenic heterozygosity contributes to arrhythmogenic right ventricular cardiomyopathy. J Am Coll Cardiol 2010;55:587-597.

32. Bhonsale A, Groeneweg JA, James CA, et al. Impact of genotype on clinical course in arrhythmogenic right ventricular dysplasia/cardiomyopathyassociated mutation carriers. Eur Heart J 2015;36:847-855.

33. Medeiros-Domingo A, Saguner AM, Magyar I, et al. Arrhythmogenic right ventricular cardiomyopathy: implications of next-generation sequencing in appropriate diagnosis. Europace, e-pub ahead of print 18 May 2016.

34. Sen-Chowdhry S, Syrris P, Prasad SK, et al. Left-dominant arrhythmogenic cardiomyopathy. an under-recognized clinical entity. J Am Coll Cardiol 2008:52:2175-2187.

35. Murray MF. Your DNA is not your diagnosis: getting diagnoses right following secondary genomic findings. Genet Med 2015;18:1-3.

36. Quarta G, Muir A, Pantazis A, et al. Familial evaluation in arrhythmogenic right ventricular cardiomyopathy: impact of genetics and revised task force criteria. Circulation 2011;123:2701-2709. 